\title{
Observation of Cascaded Two-Photon-Induced Transitions between Fluxoid States of a SQUID
}

\author{
Siyuan Han \\ Department of Physics and Astronomy, University of Kansas, Lawrence, Kansas 66045
}

R. Rouse

Sun Microsystems, Sunnyvale, California 94086

J.E. Lukens

Department of Physics and Astronomy, University at Stony Brook, Stony Brook, New York 11794-3800

(Received 21 September 1999)

\begin{abstract}
We present evidence for transitions between fluxoid wells of a SQUID due to cascaded, two-photon processes. Such transitions are evidenced by an anomalous dependence on the transition rate from the one-photon resonant level within the initial well, which cannot be explained by previously observed macroscopic resonant tunneling. These two-photon processes may be a significant source of decoherence in SQUID qubits subject to microwave radiation.
\end{abstract}

PACS numbers: 74.50. $+\mathrm{r}, 03.65 .-\mathrm{w}, 85.25 . \mathrm{Dq}$

The degree to which macroscopic degrees of freedom (MDFs) obey quantum mechanics is a perennial source of paradoxes and debates [1-4]. Examples of these MDFs include the center of mass of a baseball, the magnetization vector of a solid, and (the focus here) the phase difference $\varphi$ of the superconducting wave function across a Josephson junction or (equivalently) the magnetic flux $\Phi$ enclosed by a SQUID. $\varphi$ and $\Phi$ typically represent the collective motion of a large number $\left(\sim 10^{10}\right)$ of Cooper pairs, putting them well into the "macroscopic" regime. In recent years, it has been predicted theoretically and verified experimentally that under appropriate conditions, such as low temperature and weak damping, at least some aspects of the behavior of MDFs must be described quantum mechanically [5-19]. However, the existence of a coherent superposition of states of MDFs remains largely untested. Some of the most remarkable macroscopic quantum effects, such as energy level quantization, resonant tunneling, and resonant photon assisted tunneling between macroscopically distinct levels, have been observed in Josephson junctions and SQUIDs [16,20-22]. Results from SQUID experiments have shown that SQUIDs can, in many respects, be custom designed quantum elements, which are promising for applications in fundamental scientific research (e.g., tests of macroscopic quantum coherence and macrorealism) as well as potential technological applications (e.g., qubits for quantum computing). The interaction between SQUIDs and microwave fields in the quantum regime plays an important role in many of these potential applications [23] but has only just begun to be tested. In this Letter, we report the first observation of the effect of twophoton processes on the transitions of a SQUID between its macroscopically distinct fluxoid states.

The details of the SQUID system have been reported elsewhere [24]. We summarize its key features here. Figure 1 shows the schematic and equivalent potential of a SQUID biased with an applied flux (in units of the flux quantum $\Phi_{0}$ ) of $\phi_{x} \approx \frac{1}{2}$. The two wells of the potential represent the $f=0$ and 1 fluxoid state of the SQUID, which for the parameters of our experiment, have counter circulating currents with magnitudes greater than $2 \mu \mathrm{A}$. The energy levels are calculated, neglecting damping, by numerical solution of Schrödinger's equation. The effect of damping on the energy of a level is of second order $[25,26]$ and should be negligible in the low damping limit appropriate here. For $\phi_{x}=\frac{1}{2}$, the potential is symmetric with an energy barrier $\Delta U_{0}$. As $\phi_{x}$ is varied about $\frac{1}{2}$, there is an offset $\varepsilon$ in the energy minima of the wells, which is nearly linear in $\phi_{x}$. This, in turn, permits a controlled shift of the relative energy of the levels in different wells. These levels (indexed by $[f, i]$ ) with energies $E_{f, i}$ well below the barrier are localized in either the $f=0$ or $f=1$ fluxoid state while levels with energy close to and above the top of the barrier are delocalized [27]. All energies are measured with respect to $E_{0,0}$ - the energy

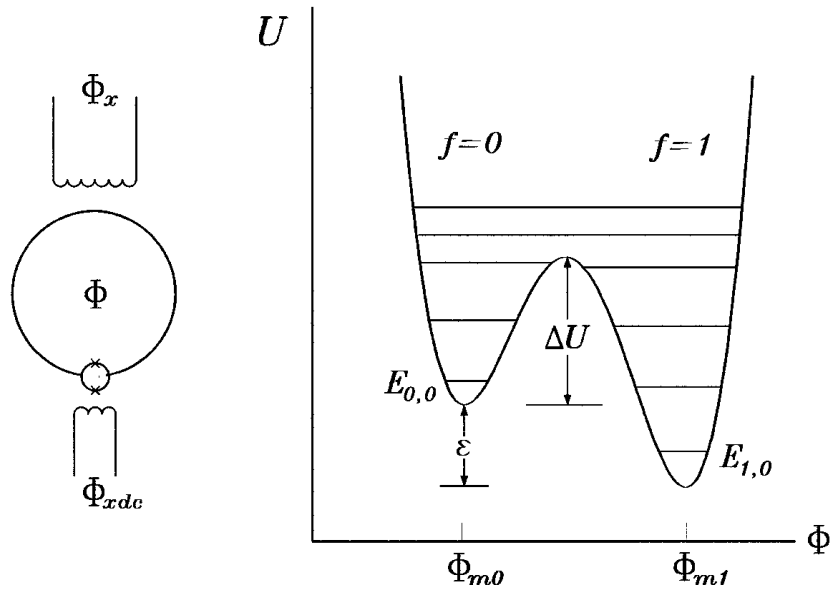

FIG. 1. Circuit schematic (left) and equivalent potential (right) describing the variable $\beta_{L}$ SQUID. 
of the lowest eigenstate of the $f=0$ well, $[0,0]$ — which we always take to be the initial state of the system. We also take $\phi_{x}$ such that $E_{0,0} \geq E_{1,0}$. The small, doublejunction loop seen in Fig. 1 acts, to a good approximation, as a single junction whose critical current $I_{c}$ can be modulated by changing the flux $\phi_{x d c}$ through the loop. We express $I_{c}$ in dimensionless units using the parameter $\beta_{L}=$ $2 \pi L I_{c} / \Phi_{0} \cdot \beta_{L}\left(\phi_{x d c}\right) \simeq \beta_{L 0}\left|\cos \left(\pi \phi_{x d c}\right)\right|$, where $\beta_{L 0}$ is just the maximum value of $\beta_{L}$, obtained for $\phi_{x d c}=0$. Thus the energy level spectrum $\left\{E_{f, i}\right\}$ of the SQUID can be varied by adjusting, in situ, $\varepsilon$ and/or $\beta_{L}$.

Measurements of the transition rate $\Gamma$ vs $\varepsilon$ of the system from the upper $(f=0)$ to lower $(f=1)$ well in the presence of a small microwave magnetic field $(\nu \approx 100 \mathrm{GHz})$ exhibit a series of resonances whose positions in $\varepsilon$ and $\beta_{L}$ agree very well with those obtained from the calculated energy levels only using four adjustable parameters $\left(L=231 \pm 4 \mathrm{pH}, C=77 \pm 3 \mathrm{fF}, \beta_{L 0}=2.01 \pm\right.$ 0.005 , and $\nu=100.8 \pm 0.5 \mathrm{GHz})$ to describe the more than 500 peaks observed. These fitting parameters, including the SQUID inductance $L$ and the junctions' capacitance $C$, are all in agreement with independently determined values.

We have previously identified two dominant transition processes for this system. One, which we call photon induced tunneling, occurs when the energy difference between a level in the $f=1$ well and the initial state $[0,0]$ equals the photon energy of the microwave field, i.e., $E_{1, j}-E_{0,0}=h \nu$. The rates for this process can be obtained from Fermi's golden rule:

$$
W_{i \rightarrow j}^{p}=\frac{2 \pi}{\hbar}\left|\hat{V}_{i j}\right|^{2} \rho\left(E_{i}-E_{j} \pm \hbar \omega\right),
$$

where $\hat{V}=\left(\Phi_{0}^{2} / 2 L\right) \phi_{x r f} \hat{\phi}, \hat{\phi}=\hat{\Phi} / \Phi_{0}$, and $\phi_{x r f}=$ $\Phi_{x r f} / \Phi_{0}$. Here and below, we use $i$ to indicate levels in the $f=0$ well and $j$ for levels in the $f=1$ well or above the barrier. This equation also describes pumping to excited levels within the initial well (replacing $j$ by $i^{\prime}$ ). The second process involves tunneling, at constant energy, between the wells at a rate given by

$$
W_{i \rightarrow j}^{t}=\frac{\left|T_{i j}\right|^{2}}{2 \hbar} \frac{\gamma_{i j} / 2}{\Delta E_{i j}^{2}+\left(\gamma_{i j} / 2\right)^{2}},
$$

where, $T_{i j}$ is the tunneling matrix element between $|i\rangle$ and $|j\rangle, \gamma_{i j}=\gamma_{i}+\gamma_{j}$ is the combined linewidth of the levels involved, and $\Delta E_{i j}$ is the energy difference between the levels. The total tunneling rate from a level is just

$$
\Gamma_{i}^{t}=\sum_{j} W_{i \rightarrow j}^{t} n_{i}
$$

where $n_{i}$ is the occupation of the initial state. This process has two resonances: One, which we call macroscopic resonant tunneling, occurs when $\Delta E_{i j}=0$ for some $j$, though, in practice, only the final state with the minimum $\Delta E_{i j}$ contributes significantly to this process. The second, which is called resonantly activated tunneling (RAT), occurs when a level in the initial well (for our system $[0,3]$ ) is in resonance so that $n_{0,3}$ is a maximum, i.e., for $\varepsilon\left(\beta_{L}\right)$ such that $E_{0,3}-E_{0,0}=h \nu$. One expects the amplitude of this RAT resonance to vary periodically with $\beta_{L}$, having a series of local maxima for values of $\beta_{L}$ such that the resonant tunneling condition is simultaneously satisfied.

The dissipation in the system, which we model as a resistive shunt $R$ across the junction, enters these rates through the linewidths $\gamma$ or the related density of states

$$
\rho(\Delta E)=\frac{\gamma}{2 \pi} \frac{1}{\Delta E^{2}+\gamma^{2} / 4} .
$$

$\gamma$ is determined for all active levels (except $[0,0]$ ) by spontaneous decay to the next lower level in the same well at a rate given by

$$
W_{i \rightarrow i^{\prime}}^{s}=\frac{2 \pi \Delta E_{i i^{\prime}}}{\hbar} \frac{R_{Q}}{R}\left|\phi_{i i^{\prime}}\right|^{2}\left[1+\operatorname{coth}\left(\frac{\Delta E_{i i^{\prime}}}{2 k_{B} T}\right)\right],
$$

where, $\Delta E_{i i^{\prime}}=E_{i}-E_{i^{\prime}}, \quad R_{Q}=h / 4 e^{2}, \quad$ and $\phi_{i i^{\prime}}=$ $\left\langle i|\hat{\phi}| i^{\prime}\right\rangle$. Since the intrawell level spacing of about $1.5 \mathrm{~K}$ is much greater than the temperature $(T \approx 30 \mathrm{~K})$, the inverse process is negligible.

In general, it is necessary to solve the master equation to obtain the occupations $n_{i}$. In fact, for most of the parameter space studied, only two initial levels are important for interwell transitions. Since the microwave perturbation is weak and the tunneling rate small, $n_{0,0} \approx 1$. The system then undergoes photoexcitation from this level to a level near resonance in either of the wells $\left(\Gamma^{p}\right)$. The excitation within the same well (to $[0,3])$ and subsequent sequential decay $(i \rightarrow i-1)$ back to $[0,0]$ results in roughly equal populations for levels $[0,3]$ through $[0,1]$, ranging from about $10^{-6}$ to $10^{-3}$ for the data depending on how close to RAT resonance (as a function of $\varepsilon$ and $\beta_{L}$ ) the system is. While we generally use a rather large set of states for the solution of the master equation, a very good estimate of $n_{i}$ in the system can be obtained from the reduced master equation $W_{0,0 \rightarrow 0,3}^{p}=n_{0,3} W_{0,3 \rightarrow 0,2}^{s}$ together with arguments above. All other decay channels for the [0,3] levels, including stimulated emission and tunneling, give a rate orders of magnitude less than that due to Eq. (5). Since $\left|T_{i j}\right|$ decreases roughly exponentially with barrier height, only the $[0,3]$ level contributes a significant tunneling rate given by Eq. (3).

A subset of the data for interwell transition probabilities (which can be directly converted to $\Gamma$ ) showing these various resonances is seen in Fig. 2, where the oscillation in the RAT amplitude (the sequence of peaks running diagonally across the graph) in $\beta_{L}$ is clearly seen. These data were obtained by varying $\varepsilon$ at a constant rate [28] while monitoring the flux $\phi$ through the SQUID with a magnetometer. The change in the magnetometer output, $\Delta \phi \cong \Delta \phi_{m} \equiv \phi_{m 1}-\phi_{m 0}$ (see Fig. 1), upon a transition of fluxoid states was used to trigger a sampler, which stored the corresponding value of $\varepsilon$. This process was repeated 6000 times for a given $\beta_{L}$ to obtain the transition probability histograms in Fig. 2. The focus of the remainder of this Letter is the deviation of the amplitudes of these 


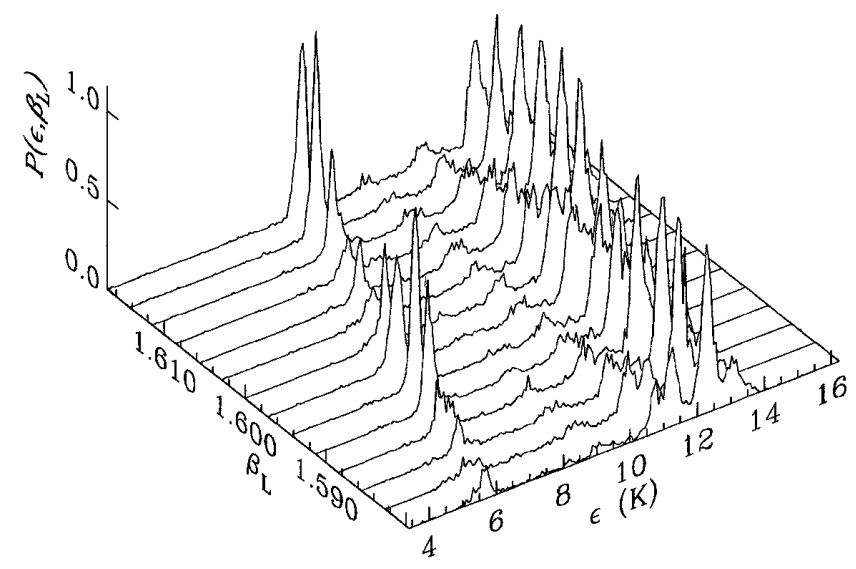

FIG. 2. The relative probabilities $P$ for transitions from the $f=0 \rightarrow 1$ fluxoid wells as the potential tilt $\varepsilon$ is increased at a constant rate for a range of critical currents $\left(\beta_{L}\right)$. The sequence of peaks running diagonally across the graph is of RAT resonances, while those roughly parallel to the $\beta_{L}$ axis correspond to macroscopic resonant tunneling and photoinduced transition processes.

RAT peaks in other regions of the parameter space from those expected on the basis of the single photon processes discussed above.

Figure 3 (top) shows the calculated level structure of the system as a function of $\beta_{L}$ for $\varepsilon$ such that the system is on the RAT peak. The predicted $\varepsilon\left(\beta_{L}\right)$ dependence for the RAT peak, along with the measured peak locations, is shown in Fig. 3 (bottom). One notes (Fig. 3), that the tunnel barrier seen from $[0,3]$ at the RAT peak increases with decreasing $\beta_{L}$ even though the barrier for $\varepsilon=0, \Delta U_{0}$, is decreasing with $\beta_{L}$. Since $T_{i j}$ at the RAT peak decreases exponentially with decreasing $\beta_{L}$, the overall rate predicted by Eq. (3) decreases rapidly for small $\beta_{L}$. Figure 4 shows the measured amplitude of the RAT peaks (dots) along with those predicted considering only the single photon processes discussed above (dashed line) $[29,30]$. As can be seen, the agreement is very good in the large $\beta_{L}$, high rate, region but fails completely for small $\beta_{L}$. Not only are the predicted rates much lower than observed, but the locations of the predicted maxima are actually closer to those of the measured minima. This strongly suggests that, as the tunneling rate decreases, a new process is taking over as the dominant mechanism for interwell transitions.

A clue to the nature of this new process is found in the level diagram in Fig. 3 (top). Here, the dashed and dash-dotted lines indicate the energies $h \nu$ above the [0,3] and $[0,2]$ levels, respectively. The positions of the observed maxima in the RAT amplitudes for small $\beta_{L}$ coincide with the intersection of these energies with delocalized levels lying above the barrier, indicating that these levels serve as second intermediate levels (SIL) for the new process. The solution of the master equation including the levels up to $3 h \nu$ shows that, on these resonances, the occupation of the states above $[0,3]$ up to the second intermediate

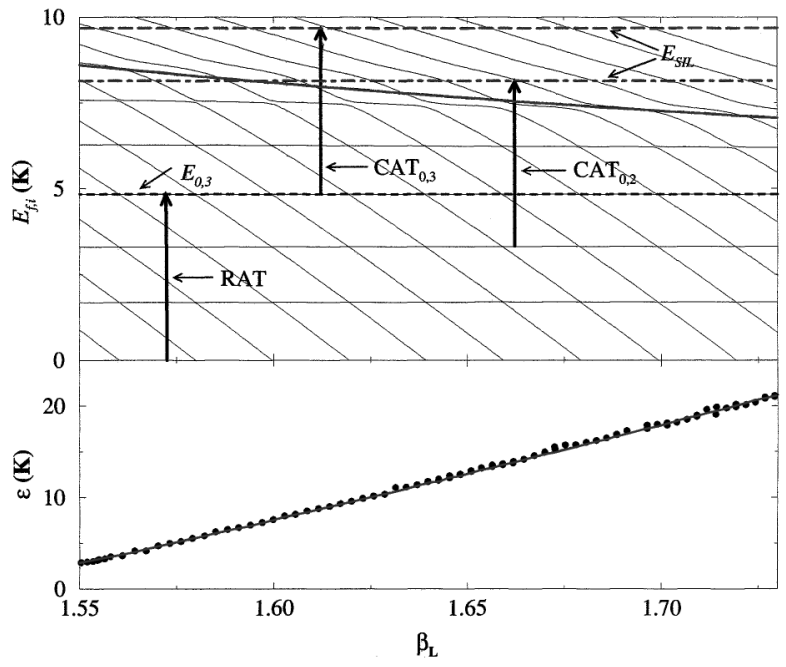

FIG. 3. Top: The calculated level structure of the system as a function of $\beta_{L}$ for $\varepsilon$ such that the system is on the RAT resonance $\left(E_{0,3}=h \nu\right)$. The heavy arrows indicate various photon absorption processes (see text) contributing to interwell transitions. The heavy solid line shows the position of the tunnel barrier, while the intersections of the upper dashed and dash-dotted lines $\left(E_{\mathrm{SIL}}\right)$ with an energy level (lighter solid lines) indicate resonances due to cascaded photon absorption. Bottom: Measured (dots) vs calculated (solid line) position of peaks corresponding the RAT resonances.

level increases significantly reaching $n_{\mathrm{SIL}} \approx 10^{-8}$ on resonance. Since the system, once excited above the barrier, will undergo sequential decay with roughly a $50 \%$ probability of reaching the $f=1$ well, these cascade activated transitions (CATs) involving the sequential absorption of two photons can easily give a rate much greater than that

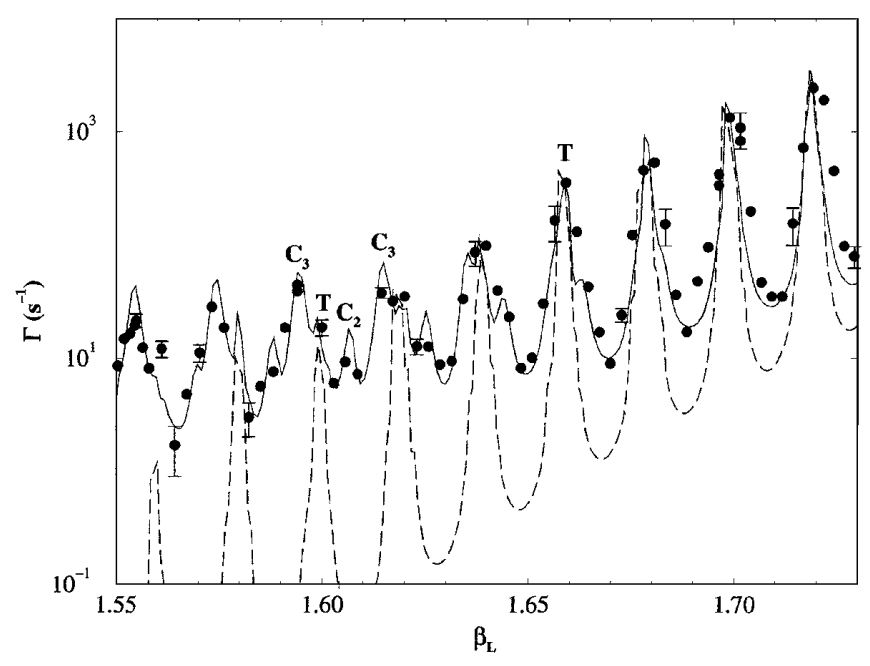

FIG. 4. Measured amplitude of the RAT resonance peaks (dots) with representative error bars compared with the calculated amplitudes including only single photon absorption (dashed line) and cascaded two-photon absorption (solid line). Several of the calculated peaks are identified with their corresponding processes by $T$ (tunneling from $[0,3]$ ) or $C$ (cascaded two-photon absorptions to a second resonant level above the barrier). 
produced by Eq. (3) in the small $\beta_{L}$ region. The solid curve in Fig. 4 shows the calculated transition rate including these CAT transitions (again with Gaussian smearing). Indexing the CAT processes by the initial state for the second photon absorption, the calculations show significant peaks for $\mathrm{CAT}_{0,3}$ and $\mathrm{CAT}_{0,2}$. The peaks in this calculated rate are labeled $T$ (for single photon tunneling transitions-RAT) and $\mathrm{C}_{3}$ and $\mathrm{C}_{2}$ for the $\mathrm{CAT}_{0,3}$ and $\mathrm{CAT}_{0,2}$ cascade transitions, respectively. The second intermediate level for the $\mathrm{CAT}_{0,1}$ transition lies below the barrier, and so does not give a significant transition rate. Also, the second intermediate level for the $\mathrm{CAT}_{0,2}$ transition drops below the barrier for $\beta_{L}<1.59$ (see Fig. 3), accounting for the rapid decrease in the amplitude of this peak relative to the $\mathrm{CAT}_{0,3}$ peak for the smallest $\beta_{L}$. As can be seen, the data agree within experimental error with these calculated rates including cascaded two-photon processes, in marked contrast to the evident deviation from the predictions including only single photon absorption.

The calculated rates depend on two unknown parameters, the damping $R$ and the rf flux amplitude $\phi_{x r f}$. These have been used as fitting parameters with optimum values of $R=5 R_{Q}$ and $\phi_{x r f}=0.001$. We estimate the uncertainty in these parameters to be about $\pm 50 \%$. This value for the damping compares favorably with the value of $R=5.3 R_{Q}$ corresponding to the Lorentzian linewidth obtained from the deconvolution of one of the strongest RAT peaks (near $\beta_{L}=1.70$ ) into its Lorentzian (intrinsic) and Gaussian (measurement noise) components [24]. This corresponds to a damping $\alpha \equiv \Delta \phi_{m}^{2} \frac{R_{Q}}{R}$ of 0.03 putting the system well within the regime $(\alpha<0.5)$ where macroscopic quantum coherence (MQC) is possible.

In conclusion, we have observed anomalies in the transition rates between the fluxoid states of SQUIDs in microwave magnetic fields occurring when the parameters $\varepsilon$ and $\beta_{L}$ of the potential are such that a state within the initial well is resonant with the microwave photons. The transition rates measured at the resonant peaks (RAT) corresponding to these conditions agree well with those calculated including cascaded two-photon absorption by the system. In particular, for low critical current $\left(\beta_{L}\right)$, where tunneling involving single photon absorption is suppressed, the measured peak amplitudes show local maxima where a second intermediate level has an energy $h \nu$ above the initial resonant level. These data provide the first confirmation of such two-photon transitions in SQUIDs, and indicate the importance of considering these processes as potential sources of decoherence in SQUID qubits.

The authors are grateful for many helpful discussions with D. Averin, A. Garg, and K. Likharev. This work was supported in part by AFOSR No. F49620-99-1-0205, the State of Kansas No. S99041, and NSF No. DMR9876874 (Kansas), and by NSF No. DMR9876850 and ARO No. DAAD19-99-1-0341 (Stony Brook).
[1] E. Schrödinger, Naturwissenschaften 23, 844 (1935).

[2] A. Leggett, Contemp. Phys. 25, 583 (1984).

[3] A. Leggett and A. Garg, Phys. Rev. Lett. 54, 857 (1985).

[4] L. Ballentine, Phys. Rev. Lett. 59, 1493 (1987).

[5] A. Caldeira and A. Leggett, Phys. Rev. Lett. 46, 211 (1981).

[6] S. Chakravarty, Phys. Rev. Lett. 49, 681 (1982).

[7] S. Chakravarty and S. Kivelson, Phys. Rev. Lett. 50, 1811 (1983).

[8] S. Chakravarty and A. J. Leggett, Phys. Rev. Lett. 52, 5 (1984).

[9] M.P. Fisher and A. Dorsey, Phys. Rev. Lett. 54, 1609 (1985).

[10] A. Leggett et al., Rev. Mod. Phys. 59, 1 (1987).

[11] W. Zwerger, Z. Phys. B 53, (1983).

[12] U. Weiss, H. Grabert, and S. Linkwitz, J. Low Temp. Phys. 68, 213 (1987).

[13] E. Chudnovsky and L. Gunther, Phys. Rev. Lett. 60, 661 (1988).

[14] R. F. Voss and R. A. Webb, Phys. Rev. Lett. 47, 265 (1981).

[15] S. Washburn, R. Webb, R. Voss, and S. Faris, Phys. Rev. Lett. 54, 2712 (1985).

[16] J. M. Martinis, M. H. Devoret, and J. Clarke, Phys. Rev. Lett. 55, 1543 (1985).

[17] D. Schwartz, B. Sen, C. Archie, and J. Lukens, Phys. Rev. Lett. 55, 1547 (1985).

[18] M. H. Devoret, J.M. Martinis, and J. Clarke, Phys. Rev. Lett. 55, 1908 (1985).

[19] S. Han, J. Lapointe, and J. Lukens, Phys. Rev. Lett. 66, 810 (1991).

[20] R. Rouse, S. Han, and J. Lukens, Phys. Rev. Lett. 75, 1614 (1995).

[21] S. Han, R. Rouse, and J. Lukens, Phys. Rev. Lett. 76, 3404 (1996).

[22] P. Silvestrini, V. Palmieri, B. Ruggiero, and M. Russo, Phys. Rev. Lett. 79, 3046 (1997).

[23] S. Lloyd, Science 261, 1569 (1993).

[24] R. Rouse, S. Han, and J. Lukens, in Phenomenology of Unification from Present to Future, edited by G. D. Palazzi, C. Cosmelli, and L. Zanello (World Scientific, Singapore, 1998), pp. 207-224.

[25] W. Bialek, S. Chakravarty, and S. Kivelson, Phys. Rev. B 35, 120 (1987).

[26] J. Schmidt, A. Cleland, and J. Clarke, Phys. Rev. B 43, 229 (1991).

[27] A delocalization also occurs for values of $\varepsilon$ such that $E_{0, i}=E_{1, j}$. However, this effect is not important for the results presented here, since it occurs for a range of $\varepsilon$ much less than the instrumental smearing of $\varepsilon$, as discussed in the text.

[28] The rate of change of $\Phi$ was always sufficiently slow $\left(<1 \Phi_{0} \mathrm{~s}^{-1}\right)$ that the sample is well into the adiabatic regime.

[29] Note that the photon-induced transition process is included in this theory curve but contributes a maximum of $10 \%$ (and usually much less) to these amplitudes.

[30] These calculated rates include a Gaussian smearing with FWHM of $2 \times 10^{-4}$ in $\phi_{x}$ to account for the measured effect of the magnetometer noise on the trigger circuit, which samples $\phi_{x}$ when a fluxoid transition occurs. 